\title{
Optimization of Epidemic router by new forwarding queue mode TSMF
}

\author{
Qaisar Ayub, Sulma Rashid, Dr.Mohd Soperi bin Mohd Zahid \\ Faculty of Computer Science \& Information System, \\ Department of Computer System \& Communication \\ Universiti Teknologi Malaysia (UTM) \\ Skudai - Johor, 81310, Malaysia.
}

\begin{abstract}
In most of dynamic Ad hoc sensor wireless applications ( e.g. military networks, vehicular ad hoc networks, wild life tracking sensor network), it is not possible to sustain an uninterrupted path from source to destination. Hence the traditional routing strategies (TCP/IP) cannot be deployed as they have to establish complete path before transmission.

DTN (disruption-tolerant network) has emerged as technology which enables the communication by intermittently connected nodes. A node in DTN may not able to transmit all messages from its forwarding queue due to limited transmission duration, dynamic topology changes and network partitioning. Therefore, the order in which the messages are forwarded becomes very important.

In this paper we propose a new message forwarding queue mode to optimize the performance of Epidemic router in terms of delivery probability. This technique is called as Transmit smallest message first (TSMF). Through simulations we prove that proposed queue mode (TSMF) out performs well as compared to existing FIFO and RANDOM.
\end{abstract}

\section{General Terms}

Algorithm, Routing.

\section{Keywords}

Disruption Tolerant Networks, Epidemic router, Forwarding queue mode.

\section{INTRODUCTION}

The conventional networking architecture (TCP/IP, DNS, etc. [1], [2]) work with assumption that end to end path exist from source to destination and is not able to cope with modern networking applications such as deep space communication[8], digital content delivery in rural areas with under-development architecture[7, 3] and wild life and habit monitoring [12, 13].

To enable the communication in such environments Delay Tolerance Network DTN [9], Haggle[10] architecture proposed which works under store-carry-forward paradigm where each node in the network stores the message forwarded by other nodes, carries the message while moving and then forwarded/delivered it to node(s) it encounter.
DTN routing strategies can be divided into two categories, single copy and multi copy. In single copy scheme $[14,15]$ there is only one copy of message in the whole network which is forwarded to a single path, whereas in multi copy scheme multiple copies of each message are forwarded to multiple paths which increase the probability of message delivery. One example of multi copy is epidemic routing [11], where a node floods the message to entire network to increase delivery probability. Many variation of epidemic routing schemes have been proposed such as spray\&wait , K-copy, Multi-copy [4,5,6] and Probabilistic forwarding[16].In Contrast to single copy schemes ,multi copy schemes gain more benefit from delivery performance, (lower latency average) at the cost of more transmission over head and buffer occupancy.

Due to finite bandwidth and sudden intermission, DTN node with Epidemic may not be able to transmit all messages from its forwarding queue. In such circumstances, the order how the message is forwarded to next encounter node become extremely important [17].

In this paper we studied the impact of packet forwarding order with Epidemic routing protocol and optimize its performance in terms of delivery probability by introducing a new message forwarding queue mode TSMF as compared to FIFO and RANDOM.

The rest of paper is arranged as follows, Section 2 present router under observation. Section 3 discusses existing queuing forwarding policies, Section 4 depicts TSMF algorithm, and section 5 is about simulation setup and results with conclusion in section 6 .

\section{PROTOCOL UNDER OBSERVATION}

\subsection{Epidemic Router}

In Epidemic routing [11] each node maintains a summery vector of its buffered messages. A summery vector is an index of messages keep by nodes.

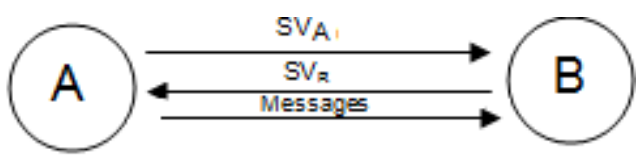

Figure 1

When two nodes A, B comes in transmission range of each other they exchange summery vectors $\left(\mathrm{SV}_{\mathrm{A}}\right.$. After this exchange each 
node can determine if other node has any message not previously received by it, in that case the node request the messages from other nodes.

\section{QUEUING MANAGEMENT POLICIES}

\subsection{First in First out (FIFO)}

In FIFO queue mode all messages are arranged according to arrival time and the message which has oldest arrival time will be transmitted first.

\subsection{Random Queue Mode (RND)}

The message is randomly selected for the transmission.

\subsection{GRTR}

"Assume A, B are nodes that meet while the destination is D, $\mathrm{P}_{(\mathrm{X}, \mathrm{Y})}$ denote the delivery predictability that a node $\mathrm{X}$ has for Destination Y. GRTR forward the message to node only if $\mathrm{P}_{\text {(B-D) }}$ $>\mathrm{P}_{\text {(A-D) }}$ " [17].

\subsection{GRTRSort}

"GRTRsort looks at difference $\mathrm{P}_{(\mathrm{B}-\mathrm{D})}-\mathrm{P}_{(\mathrm{A}-\mathrm{D})}$ values for each message between the nodes and forward the message only if $\mathrm{P}_{(\mathrm{B}-}$

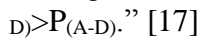

\subsection{GRTRMax}

"Select messages in descending order of $\mathrm{P}$ (B-D) forward the message only if $\mathrm{P}_{(\mathrm{B}-\mathrm{D})}>\mathrm{P}$ (A-D)." [17]

\section{ALGORITHM}

Figure 2, 3 depicts snapshot of message forwarding order with FIFO queue mode. When node-A comes in contact with node-B, A transmit its summery vector $\mathrm{SV}_{\mathrm{A}}$ to $\mathrm{B}, \mathrm{B}$ performs a logical and

Node-A

\begin{tabular}{|l|l|c|c|c|}
\hline Mid & MS & AT & TT & ATT \\
\hline M1 & $800 \mathrm{~KB}$ & $50 \mathrm{~S}$ & $8 \mathrm{~s}$ & \\
\cline { 1 - 4 } M2 & $200 \mathrm{~KB}$ & $100 \mathrm{~S}$ & $2 \mathrm{~s}$ & \multirow{2}{*}{$8 \mathrm{~s}$} \\
\cline { 1 - 3 } M3 & $100 \mathrm{~KB}$ & $150 \mathrm{~S}$ & $1 \mathrm{~s}$ & \\
\cline { 1 - 3 } M4 & $500 \mathrm{~KB}$ & $200 \mathrm{~S}$ & $5 \mathrm{~s}$ & \\
\hline
\end{tabular}

Figure 2

operation between $\mathrm{SV}_{\mathrm{A}}$ and negation of its summery vector $\left(\mathrm{SV}_{\mathrm{B}}\right)$ to find out the messages $S V_{R}$ which it needs from $A$.

$$
\mathrm{SV}_{\mathrm{R}}=\mathrm{SV}_{\mathrm{A}}+\Gamma \mathrm{SV}_{\mathrm{B}} \Rightarrow(\mathrm{M} 1, \mathrm{M} 2, \mathrm{M} 3, \mathrm{M} 4) \text {. }
$$
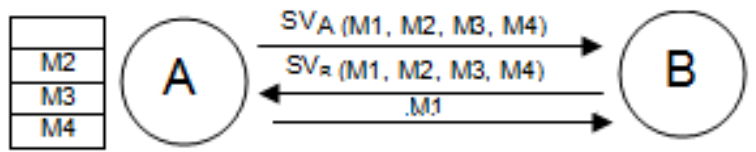

Figure 3

Node-B then transmits $\mathrm{SV}_{\mathrm{R}}$ to $\mathrm{SV}_{\mathrm{A}}$ for required message transmission. As available Transmission time is (8s) only M1 (Transmission time 8s) can be transmitted (Figure 3).

Figure 4 shows the message forwarding order with queue mode TSMF where small size messages are popped up.

Node-A

\begin{tabular}{|l|l|c|c|c|}
\hline Mid & MS & AT & TT & ATT \\
\hline M3 & $100 \mathrm{~KB}$ & $150 \mathrm{~s}$ & 1 & \multirow{1}{*}{} \\
\cline { 1 - 4 } M2 & $200 \mathrm{~KB}$ & $100 \mathrm{~s}$ & 2 & \multirow{2}{*}{$8 \mathrm{~s}$} \\
\cline { 1 - 3 } M4 & $500 \mathrm{~KB}$ & $200 \mathrm{~s}$ & 5 & \\
\cline { 1 - 3 } M1 & $800 \mathrm{~KB}$ & $50 \mathrm{~s}$ & 8 & \\
\hline
\end{tabular}

\section{Figure 4}

By available Transmission time (M3, M2, and M4) will be transmitted. Rises in message transmission increase the delivery probability (Figure 5).

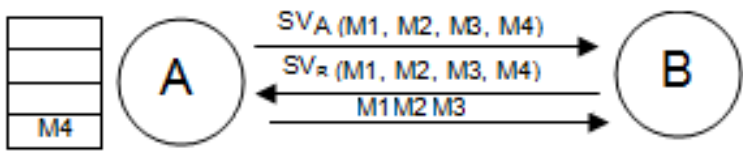

Figure 5

Algorithm

- Get Messages from Router

For each Message IN RouterMessageList

ADD (MessageLIST, message)

End loop

- Sort the MessageLIST according to size in Ascending order (TSMF)

SortedMessageList=SORT $($ MessageLIST)

- Transmit Message

For each Messages m: SortedMessageList

Send (Message)

End loop

\section{SIMULATION AND RESULTS}

In following sections we evaluate the performance of TSMF queue mode as compared to First in First out (FIFO) and Random by using DTN ONE simulator. The ONE [6 ] is a discrete event simulator written in Java. The main objective of simulator is to implement DTN store-carry-forward paradigm where the probability of disconnections and failures is high.

Table 1. Simulation parameters

\begin{tabular}{|l|l|}
\hline Number of Nodes & 126 \\
\hline Movement model 1 & Random way point \\
\hline Movement model 2 & Map Route movement \\
\hline Router & Epidemic \\
\hline Number of groups & 06 \\
\hline
\end{tabular}




\begin{tabular}{|l|l|}
\hline Message size & $500 \mathrm{~KB}$ to $1 \mathrm{MB}$ \\
\hline Transmission range & $10 \mathrm{M}$ \\
\hline Transmission speed & $250 \mathrm{KBps}$ \\
\hline Message TTL & Infinite \\
\hline Simulation area & $4500 \mathrm{~m} \times 3400 \mathrm{~m}$ \\
\hline
\end{tabular}

\subsection{Delivery probability w.r.t Time}

From Figure 6 we observe that the delivery probability of FIFO, RANDOM and TSMF queue modes. It can be seen that by increasing simulation time, TSMF has performed well in all cases due to reason of transmitting the smallest message first, while in FIFO large size message are selected and take more transmission time thus resulting in lower delivery probability.

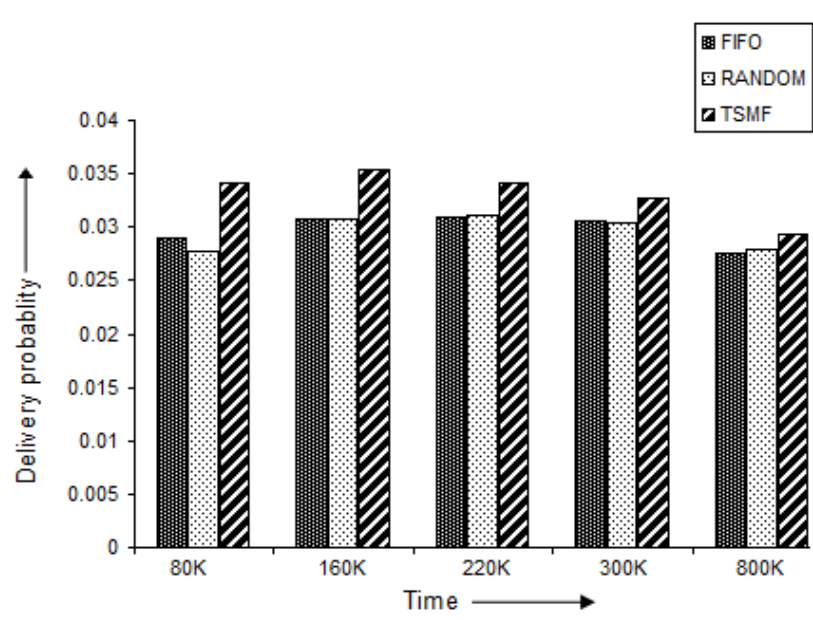

Figure 6

\subsection{Delivery probability w.r.t Buffer Sizes}

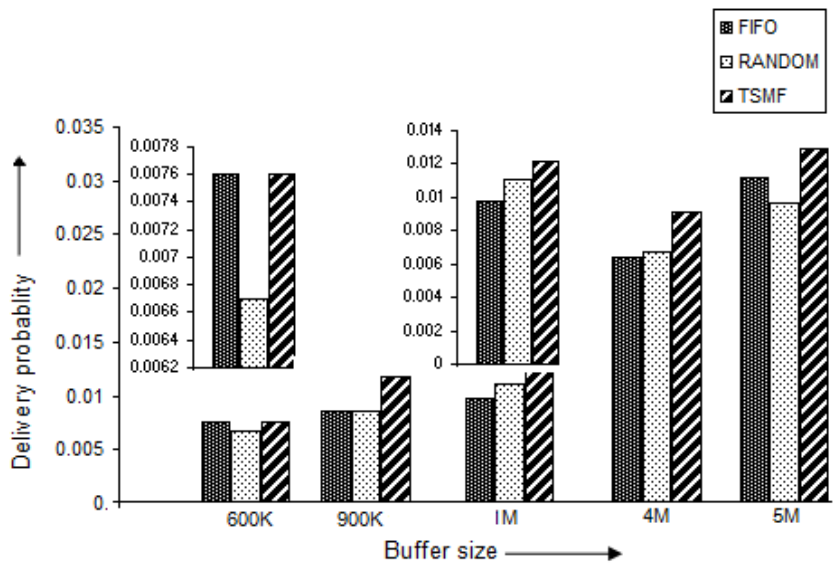

Figure 7

Figure 7 depicts the performance of FIFO, RANDOM and TSMF queue mode by shifting the buffer sizes. We can observe that by increasing buffer sizes TSMF persistently raise the delivery probability; further TSMF has also stimulated the delivery probability on small buffer size $(900 \mathrm{~K}, 1 \mathrm{M})$.

\subsection{Delivery probability w.r.t Message Creation interval}

Figure 8 simulated the activity of FIFO, RANDOM and TSMF queue mode by varying the message creation interval in seconds. Graph has depicted that TSMF has sustained the delivery probability for all ranges compared to FIFO and RANDOM.

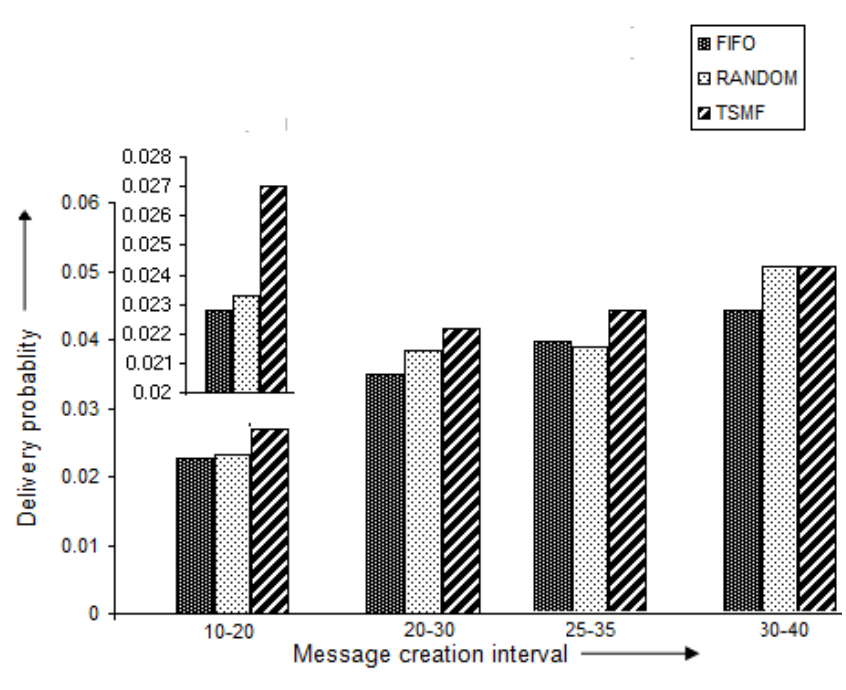

Figure 8

\subsection{Delivery probability w.r.t Group Nodes Speed}

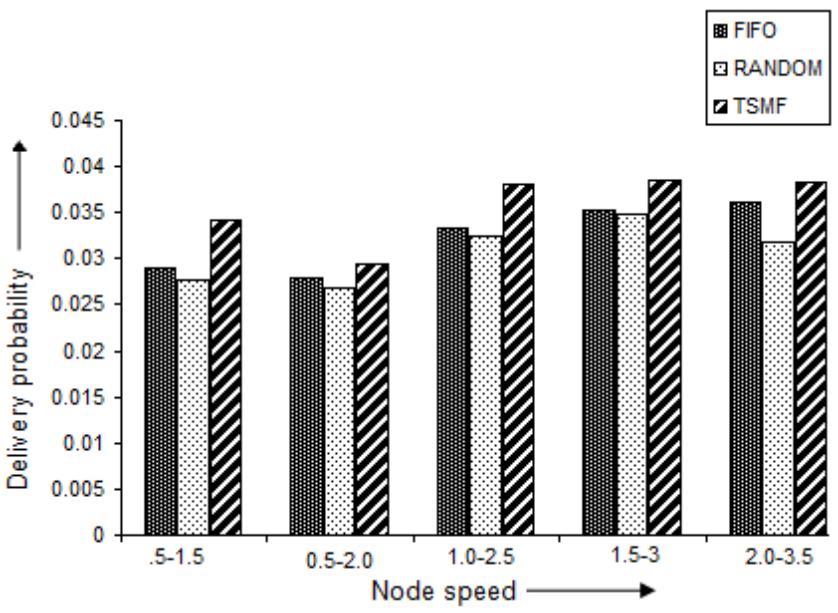

Figure 9 
In figure 9 we find out the act of FIFO, RANDOM and TSMF queue mode in terms of node speed. When nodes are moving with high speed, they have limited time to communicate with each other so in that situations forwarding mechanism becomes very important. It can be clearly seen that TSMF with transmitting smallest message first out perform well with all ranges of node speed.

\section{CONCLUSION}

In this paper we propose a new message forwarding queue mode to optimize the performance of Epidemic router in terms of delivery probability. This technique is called as Transmit smallest message first (TSMF).

\section{REFERENCES}

[1] Delay Tolerant Networking Research Group. 2007 [Online]. Available: http://www.dtnrg.org.

[2] J. Scott, P. Hui, J. Crowcroft, and C. Diot, "Haggle: A networking architecture designed around mobile users," in Proc. IFIP Conf. Wireless On-Demand Network Systems and Services (WONS), 2006.

[3] Saami network connectivity (SNC) project, March 2005. http://www.snc.sapmi.net/.

[4] T. Spyropoulos, K. Psounis, and C. S. Raghavendra." Spray and wait: an efficient routing scheme for intermittently connected mobile networks", In SIGCOMM Workshop on Delay Tolerant Networking (WDTN), 2005.

[5] T. Small and Z. J. Haas. Resource and performance tradeoffs in delay-tolerant wireless networks. In SIGCOMM Workshop on Delay Tolerant Networking (WDTN), 2005.

[6] T. Spyropoulos, K. Psounis, and C. Raghavendra. Efficient Routing in Intermittently Connected Mobile Networks: The Multi-copy Case. In ACM/IEEE Transactions on Networking, 2007.

[7] Z. J. Haas and T. Small. A New Networking Model for Biological Applications of Ad Hoc Sensor Networks. IEEE/ACM Transactions on Networking, 14, No. 1:27-40, 2006.

[8] Interplanetary internet special interest group (IPNSIG),Dec 2002. http://www.ipnsig.org.
[9] FALL, K. A Delay-Tolerant Network Architecture for Challenged Internets. In Proc. of ACM SIGCOMM (2003).

[10] SCOTT, J., HUI, P., CROWCROFT, J., AND DIOT, C. Haggle: Networking Architecture Designed Around Mobile Users. In Proceedings of IFIP WONS (2006).

[11] Vahdat and D. Becker. Epidemic Routing for Partiallyconnected Ad hoc Networks. Technical Report CS-2000-06, Duke University, July 2000.

[12] Philo Juang, Hidekazu Oki, Yong Wang, Margaret Martonosi, Li-Shiuan Peh, and Daniel Rubenstein. Energyef_cient computing for wildlife tracking: Design tradeoffs and early experiences with zebranet. In Proceedings of Tenth International Conference on Architectural Support for Programming Languages and Operating Systems (ASPLOS-X), San Jose, CA, October 2002.

[13] Tara Small and Zygmunt Haas." The shared wireless infestation model - a new ad hoc networking paradigm (or where there is a whale, there is a way)", In Proceedings of The Fourth ACM International Symposium on Mobile Ad Hoc Networking and Computing (MobiHoc 2003), pages 233.244, June 2003.

[14] KERÄNEN, A., AND OTT, J. Increasing Reality for DTN Protocol Simulations. Tech. rep., Helsinki University of Technology, Networking Laboratory, July 2007.

[15] T. Spyropoulos, K. Psounis, and C. Raghavendra A, C. $\mathrm{S}$. "Single-copy routing in intermittently connected mobile networks," IEEE/ACM Transactions on Networking (TON), vol. 16, pp. 63-76, Feb. 2008.

[16] A. Lindgren, A. Doria, and O. Schelen, "Probabilistic routing in intermittently connected networks," SIGMOBILE Mobile Computing and Communication Review, vol. 7, no. 3, 2003.pp 19-20.

[17] A.indgren and K. S. Phanse, "Evaluation of queuing policies and forwarding strategies for routing in intermittently connected networks,"in Proc. of IEEE COMSWARE, pp. 1-10, Jan. 2006. 